\title{
Gastric cancer cell proliferation is suppressed by frizzled-2 short hairpin RNA
}

\author{
MINORU TOMIZAWA ${ }^{1}$, FUMINOBU SHINOZAKI ${ }^{2}$, YASUFUMI MOTOYOSHI ${ }^{3}$, \\ TAKAO SUGIYAMA $^{4}$, SHIGENORI YAMAMOTO ${ }^{5}$ and NAOKI ISHIGE ${ }^{6}$ \\ Departments of ${ }^{1}$ Gastroenterology, ${ }^{2}$ Radiology, ${ }^{3}$ Neurology, ${ }^{4}$ Rheumatology, ${ }^{5}$ Pediatrics and ${ }^{6}$ Neurosurgery, \\ National Hospital Organization, Shimoshizu Hospital, Yotsukaido City, Chiba 284-0003, Japan
}

Received November 9, 2014; Accepted December 29, 2014

DOI: 10.3892/ijo.2015.2830

\begin{abstract}
In order to identify novel targets for the molecular therapy of gastric cancer (GC), we investigated the mRNA and protein expression of frizzled-2 (Fz2), a Wnt signaling pathway receptor. Reverse-transcriptase polymerase chain reaction (PCR) amplification was utilized to determine the expression patterns of $F z$ genes in normal stomach and in the GC cell lines MKN45 and MKN74. Immunostaining was performed on surgical specimens of GC using an antibody against Fz2. The 3-(4,5-dimethylthiazol-2-yl)-5-(3-carboxymethoxyphenyl)-2- (4-sulfophenyl)-2H-tetrazolium inner salt (MTS) assay was performed on MKN45 cells and MKN74 cells transfected with Fz2 short-hairpin (sh) RNA. Cell motility was analyzed by scratch assay following Fz2 shRNA. Real-time quantitative PCR was performed to analyze the expression levels of cyclin D1 and matrix metallopeptidase 9 (MMP-9). Fzl, 3, 6 and 8 were expressed in normal stomach, and in MKN45 and MKN74 cells. Fz2 was expressed in normal stomach and in MKN45, but not in MKN74 cells. Well-differentiated GC tissue was weakly positive for Fz2 in cell membranes. Fz2 was positive in both the cell membrane and cytoplasm of GC tissues of moderately differentiated and poorly differentiated adenocarcinoma. Signet ring cells were positive for cytoplasmic Fz2. Proliferation of MKN45 and MKN74 cells was suppressed by Fz2 shRNA, and a scratch assay demonstrated that Fz2 shRNA suppressed also MKN45 and MKN74 cell motility. Furthermore, Fz2 shRNA application led to downregulated mRNA expression of both cyclin D1 and MMP-9. Fz2, 3, 6 and 8 were expressed in normal stomach, and in MKN45 and MKN74 GC cells. Fz2 shRNA suppressed cell proliferation and motility of MKN45 and MKN74 cells, and downregulated cyclin D1 and MMP-9 expression in these GC cell lines.
\end{abstract}

Correspondence to: Dr Minoru Tomizawa, Department of Gastroenterology, National Hospital Organization Shimoshizu Hospital, 934-5 Shikawatashi, Yotsukaido City, Chiba 284-0003, Japan E-mail: nihminor-cib@umin.ac.jp

Key words: frizzled genes, real-time quantitative PCR, MTS assay, scratch assay, cyclin D1, matrix metallopeptidase

\section{Introduction}

Gastric cancer (GC) accounts for $8 \%$ of total cases and $10 \%$ of total deaths from cancer worldwide (1). Although the endoscopic approach has improved cancer diagnosis and treatment, especially of early GC, prognosis is still poor for advanced types $(2,3)$. Consequently, the development of effective molecular therapies for GC is eagerly awaited.

The Wnt pathway is involved in cell proliferation and differentiation (4). Wnt proteins bind to their receptor, frizzled (Fz), and its co-receptors, low-density lipoprotein receptor-related proteins 5 and 6 (LRP5/6), to form a complex $(5,6)$. Once Wnt binds to its receptor complex, cytoplasmic $\beta$-catenin is accumulated through inhibition of its degradation by the glycogen synthase kinase (GSK)-3 $\beta$ complex (7). $\beta$-catenin acts as a co-factor of the T-cell factor (TCF)/lymphoid enhancer factor (LEF) and activates target genes (8). Activity of the Wnt pathway is also controlled by inhibition of secreted proteins such as dickkopf homolog-3 (9). Constitutive activation of the Wnt pathway leads to abnormal cell growth and the development of GC $(10,11)$.

Wnt5a is upregulated in GC possibly due to demethylation of its promoter (12). The Fz3 and 6 receptors transduce the Wnt signal (13). Furthermore, the expression level of $\beta$-catenin has been shown to be upregulated in the MKN-45 GC cell line (14). Notably, upregulated $\beta$-catenin has been correlated with poor prognosis of patients with GC (14), and methylation of dickkopf homolog 3 has also been associated with poor prognosis of GC (15). Together, these reports clearly indicate that the Wnt pathway is activated in GC. It was, therefore, expected that inhibition of the Wnt pathway might be developed into a new molecular therapy for GC. In comparison, it has been shown that both downregulation of Fz2 and inhibitors of the Wnt pathway successfully suppress the proliferation of hepatocellular carcinoma and pancreatic cancer cells (16-19).

Therefore, we analyzed the effects of Fz2 inhibition using short hairpin RNA (shRNA) on the proliferation and motility of GC cell lines.

\section{Materials and methods}

This study was approved by the institutional ethics committee of the National Hospital Organization Shimoshizu Hospital, 
Yotsukaido City, Japan. Written informed consent was obtained by the commercial source (BioChain, Hayward, CA, USA).

Cell culture. The GC cell lines MKN45 and MKN74 were purchased from RIKEN Cell Bank (Tsukuba, Japan). Cells were cultured in Roswell Park Memorial Institute (RPMI)-1640 (Sigma, St. Louis, MO, USA) supplemented with $10 \%$ fetal bovine serum (FBS) (Life Technologies, Grand Island, NY, USA). Cell lines were cultured with $5 \%$ carbon dioxide at $37^{\circ} \mathrm{C}$ in a humidified chamber.

shRNA transfection. Cells were plated in 6-well plates (Asahi Techno Glass, Tokyo, Japan) and cultured until they reached $80 \%$ confluence, then transfected and cultured for an additional 48 h. Fz2 shRNA (OriGene, Rockville, MD, USA) was transfected into cells by using Lipofectamine LTX (Life Technologies), according to the manufacturer's instructions. Briefly, shRNA was incubated with PLUS reagent for $5 \mathrm{~min}$, after which LTX reagent was added. A 15-min incubation at room temperature ensued, and the complex was subsequently applied to the cell culture medium. An shRNA negative control was also purchased from OriGene.

$R N A$ isolation, reverse transcription (RT-), and real-time quantitative polymerase chain reaction $(P C R)$ amplification. Total RNA was isolated from native or transfected cell lines using Isogen (Nippon Gene, Tokyo, Japan), and $5 \mu \mathrm{g}$ used to generate cDNA with SuperScript III and oligo(dT) primers, as per the manufacturer's instructions (Life Technologies). Human whole stomach RNA was purchased from Clontech (Mountain View, CA, USA). PCR primers, annealing temperatures, reaction cycle numbers, and amplicon lengths are shown in Table I. PCR was performed using Taq DNA polymerase (Life Technologies), and products were subjected to analysis by gel electrophoresis in $2 \%$ agarose in $1 \mathrm{X}$ TAE (40 mM Tris-acetate/1 mM EDTA). Real-time quantitative PCR was performed using the Fast SYBR Green Master Mix (Life Technologies) and analyzed with the MiniOpticon Detection system (Bio-Rad, Hercules, CA, USA). Primer pairs for real-time quantitative PCR and the resultant product sizes were demonstrated in Table I. Real-time quantitative PCR was performed for 40 cycles with $5 \mathrm{sec}$ denaturation at $95^{\circ} \mathrm{C}$ and $5 \mathrm{sec}$ annealing/extension at $60^{\circ} \mathrm{C}$. GAPDH and RPL19 were used as internal controls of RT-PCR and real-time quantitative PCR, respectively.

Immunostaining. Serial sections were cut from surgical samples, formalin-fixed, and embedded in paraffin (BioChain). The samples: well-differentiated adenocarcinoma (56-weekold female), moderately-differentiated adenocarcinoma (61-year-old female), poorly-differentiated adenocarcinoma (74-year-old male), and signet ring cells (62-year-old male) (BioChain) were deparaffinized, autoclaved, and incubated first with hydrogen peroxide, and then with $2 \%$ normal goat serum in phosphate-buffered saline (PBS) (washing buffer) for $30 \mathrm{~min}$. After overnight incubation with a rabbit polyclonal anti-Fz2 antibody (1:5,000) (Sigma-Aldrich), specimens were rinsed with PBS and subsequently incubated with horseradish peroxidase-labeled anti-rabbit antibody (1:500) for $2 \mathrm{~h}$
(GE Healthcare, Pittsburgh PA, USA). Next, diaminobenzidine (Dako, Glostrup, Denmark) was applied to the tissue sections as a chromogen, and the nuclei were stained with hematoxylin (Muto Pure Chemicals Co., Ltd., Tokyo, Japan) for $15 \mathrm{sec}$. Specimens were observed and photographed under an AX80 microscope (Olympus, Tokyo, Japan).

Cell proliferation analysis. Cells were trypsinized, harvested, spread onto 96-well flat-bottom plates (Asahi Techno Glass) at a density of 1,000 cells per well, and incubated for $24 \mathrm{~h}$ in DMEM supplemented with $10 \%$ FBS. After culturing, cells were transfected with Fz2 shRNA for 72 h. Cell cultures were subjected to 3-(4,5-dimethylthiazol-2-yl)- 5-(3-carboxymethoxyphenyl)2-(4-sulfophenyl)-2H-tetrazolium inner salt (MTS) assays according to the manufacturer's instructions (Promega Corp., Madison, WI, USA). MTS is bio-reduced by cells into a colored formazan product that reduces absorbance at $490 \mathrm{~nm}$. Absorbance was analyzed at a wavelength of $490 \mathrm{~nm}$ with an iMark Microplate Absorbance Reader (Bio-Rad).

Scratch assay. Cells were plated on 4-well chamber slides (Becton-Dickinson, Franklin Lakes, NJ, USA). When cells reached confluence, they were scratched with $200 \mu \mathrm{l}$ pipettes, incubated for $48 \mathrm{~h}$ and stained with hematoxylin and eosin. The stained slides were observed under an AX80 microscope (Olympus). The distance of the scratched line from the growing edge of the cells was measured at five different time points.

Statistical analysis. Cell proliferation and real-time quantitative PCR data were analyzed by a one-factor analysis of variance. Statistical analysis was performed using JMP5.0J software (SAS Institute, Cary, NC, USA). A P-value of $<0.05$ was set as statistically significant.

\section{Results}

To analyze the expression patterns of $F z$ genes in normal stomach, and in MKN45 and MKN74 cells, RT-PCR was performed and the products subjected to electrophoresis (Fig. 1A). $F z 1,3,6$ and 8 were expressed in normal stomach, and in MKN45 and MKN74 cell lines. $F z 2$ was expressed in normal stomach and in MKN45, but not in MKN74 cells. This result suggested that the expression of $F z 2$ varied between the cell lines. To determine the expression levels of $F_{z} 2$ in normal stomach, and in MKN45 and MKN74 cell lines, real-time quantitative PCR was performed (Fig. 1B). The relative expression levels of $F z 2$ in MKN45 and MKN74 lines were $1.94 \pm 0.85$ and $0.48 \pm 0.11$ (mean \pm standard deviation), respectively, as compared with that in normal stomach. It was therefore confirmed that expression of $F z 2$ was dependent on the cell line analyzed.

To reveal the expression of Fz2 in GC tissues, surgical specimens were immunostained with an antibody for the Fz2 protein (Fig. 2). Well-differentiated GC was weakly positive for Fz2 staining in the cell membranes (Fig. 2A). Fz2 staining was positive in both the cell membranes and the cytoplasm of GC tissues of moderately-differentiated and poorly-differentiated adenocarcinoma (Fig. 2B and C). Signet ring cells showed positive cytoplasmic staining for Fz2 (Fig. 2D). These results suggested that $\mathrm{Fz} 2$ was present in GC tissues, and that 


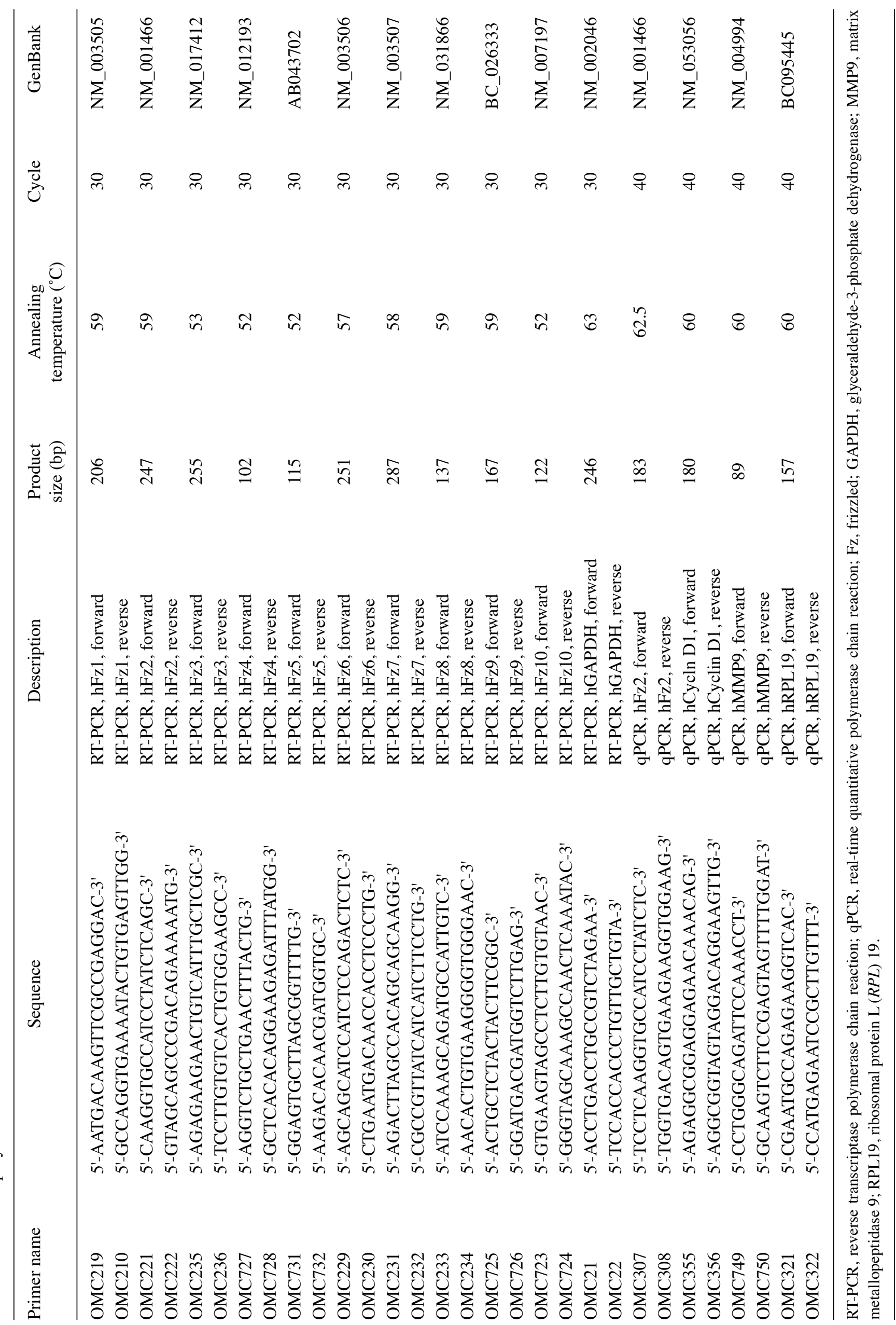




\section{A $\quad \mathrm{H} 2 \mathrm{O}$ normal $\mathrm{MKN} 45$ MKN74}
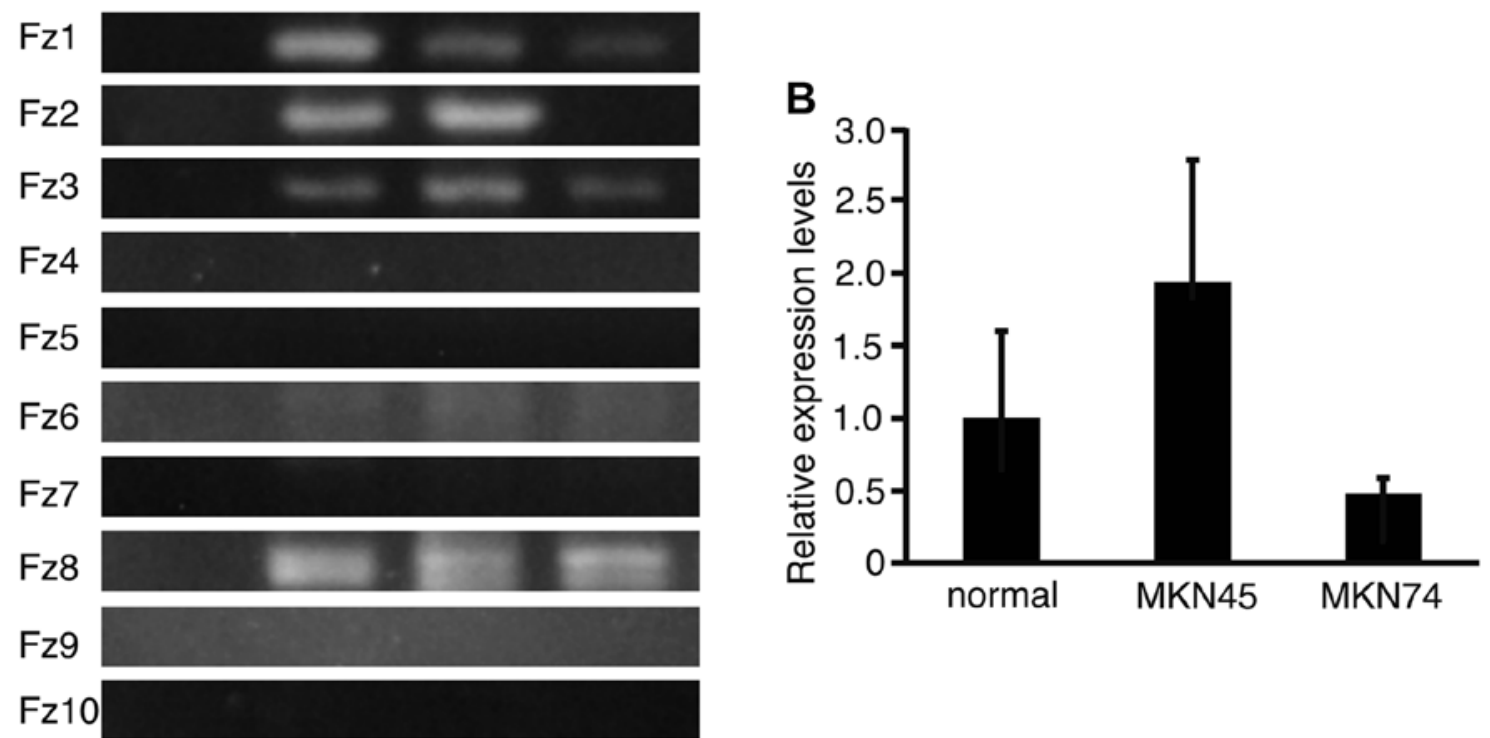

Figure 1. Expression patterns of frizzled. (A) Expression patterns of frizzled genes were analyzed using reverse-transcriptase polymerase chain reaction amplification. (B) The expression levels of frizzled-2 in normal stomach and gastric cancer cell lines were analyzed using real-time quantitative polymerase chain reaction amplification. Normal, normal stomach; error bar, standard deviation; N=3.
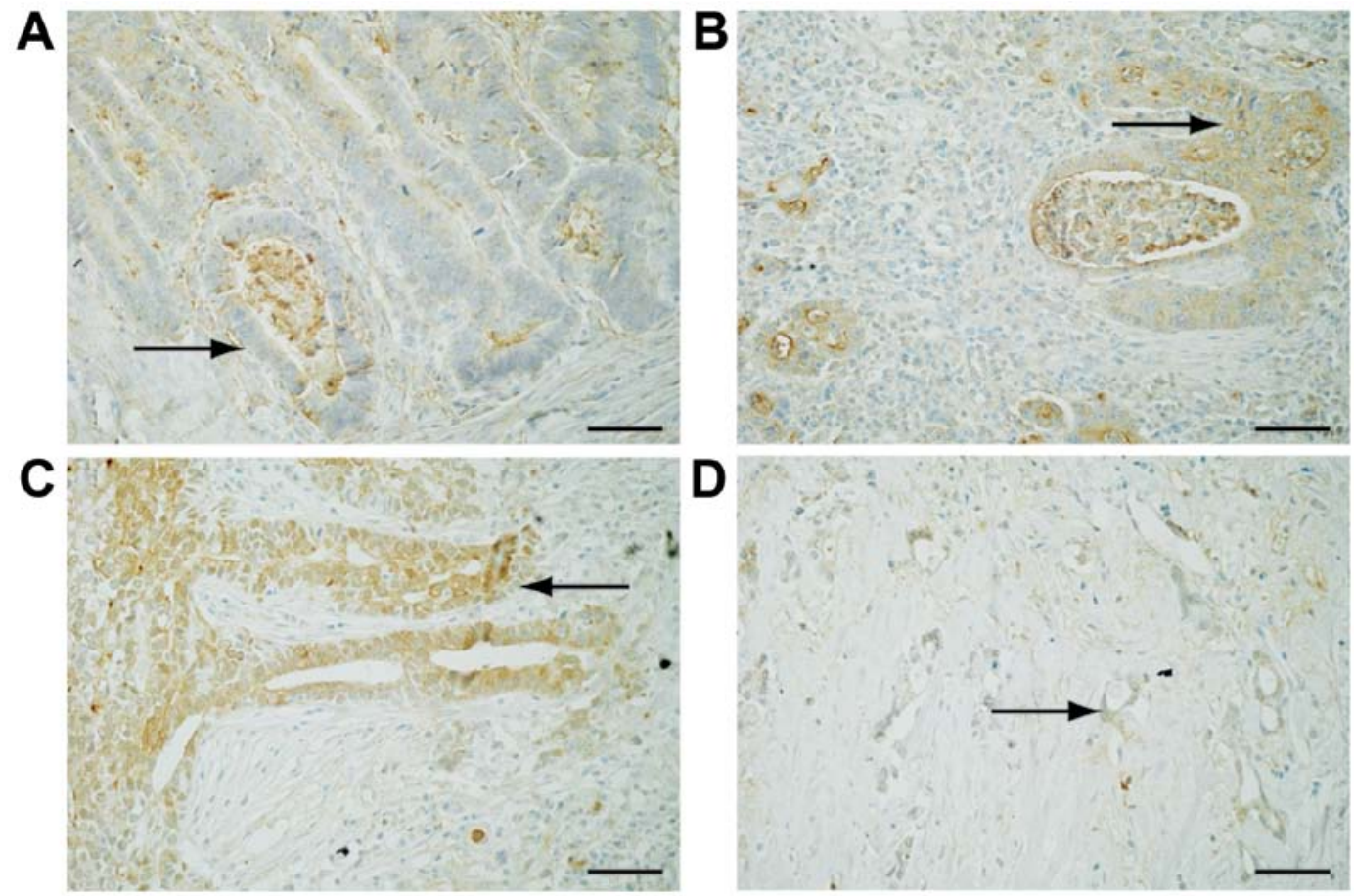

Figure 2. Expression of frizzled-2 in gastric cancer tissues and cell lines. Expression of frizzled-2 was analyzed immunohistochemically in surgical specimens of well-differentiated adenocarcinoma (A), moderately differentiated adenocarcinoma (B), poorly differentiated adenocarcinoma (C) and signet-ring cells (D). Arrows, cancer cells; original magnification, $\mathrm{x} 400$; scale bar, $50 \mu \mathrm{m}$.

its expression levels are dependent on the grade of pathological differentiation.

To address the possibility that proliferation of GC cell lines might be suppressed with downregulation of Fz2, Fz2 shRNA was transfected into the GC MKN45 and MKN74 cell lines (Fig. 3). Proliferation of the MKN45 cell line was suppressed to $53.2 \pm 23.4 \%$ of that of mock transfected cells $(\mathrm{P}<0.05)$ at
$100 \mathrm{ng} /$ well MTS (Fig. 3A); similarly, proliferation of MKN74 cells was suppressed to $59.9 \pm 38.2 \%(\mathrm{P}<0.05)$ of that of mock transfected cells at $100 \mathrm{ng} /$ well MTS (Fig. 3B).

To confirm the downregulation of Fz2 by Fz2 shRNA, the expression levels of the $F z 2$ gene were quantitated. Expression levels of cyclin D1, involved in cell proliferation (20), were also analyzed. Quantitative real-time PCR demonstrated that 

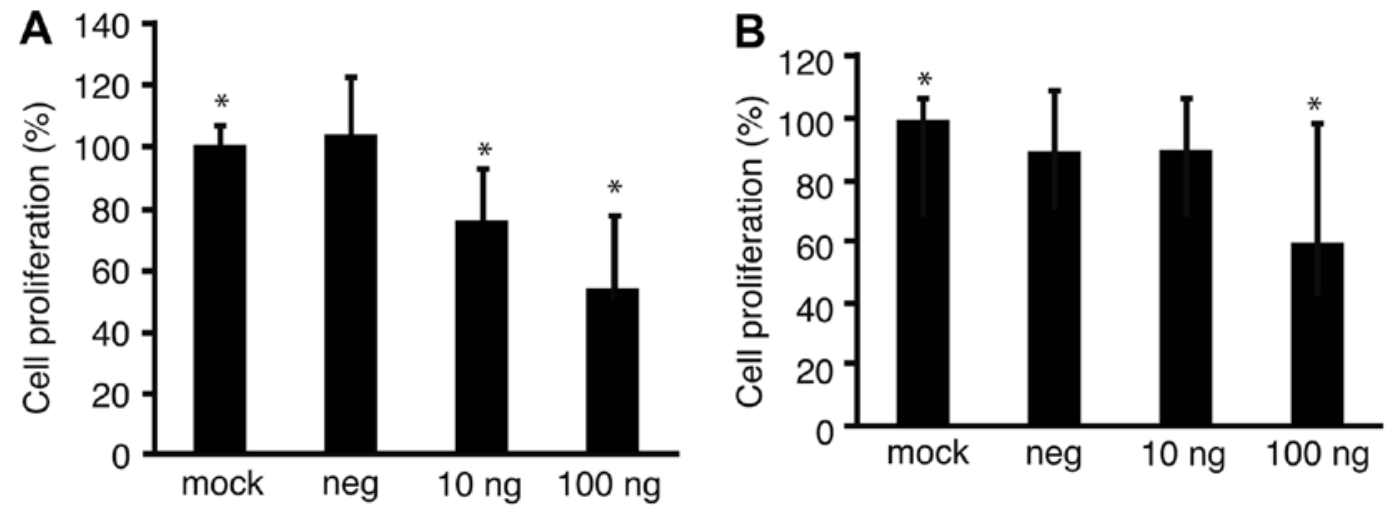

Figure 3. Cell proliferation assay. Cell proliferation was analyzed in MKN45 (A) and MKN74 (B) cells by using the 3-(4,5-dimethylthiazol-2-yl)-5-(3carboxymethoxyphenyl)-2-(4-sulfophenyl)-2H-tetrazolium inner salt (MTS) assay $72 \mathrm{~h}$ after transfection of Fz2 shRNA. Mock, mock transfection; neg, negative control; " $\mathrm{P}<0.05$ (one-factor analysis of variance); error bar, standard deviation; $\mathrm{N}=3$.
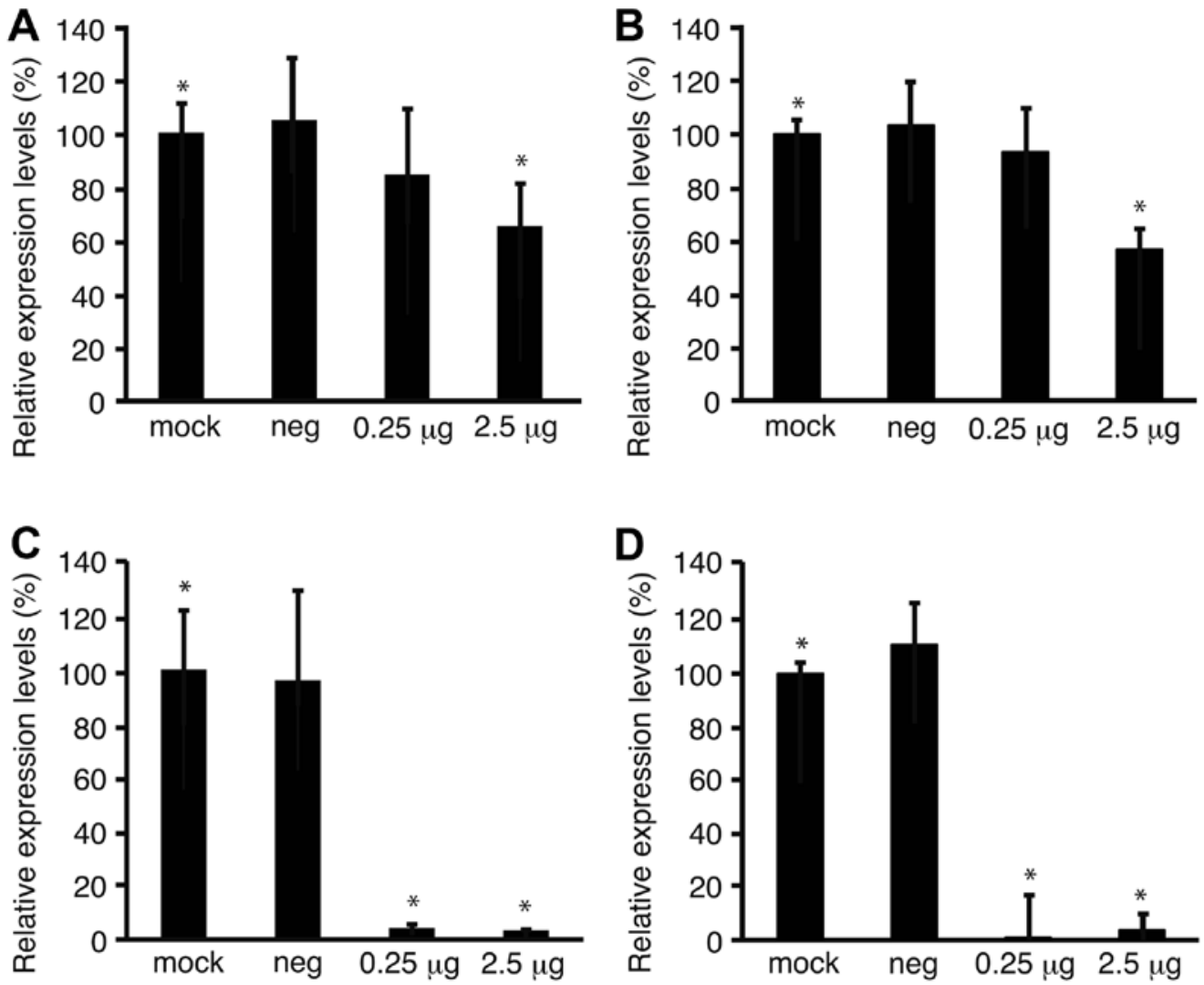

Figure 4. Expression levels of cyclin D1. Expression levels of $F z 2$ (A and C) and cyclin D1 (B and D) were analyzed by quantitative real-time polymerase chain reaction (PCR) in MKN45 (A and B) and MKN74 cells (C and D) transfected with Fz2 shRNA. Mock, mock transfected; neg, transfected with negative control; $0.25 \mu \mathrm{g}$, transfected with $0.25 \mu \mathrm{g}$ shRNA; $2.5 \mu \mathrm{g}$, transfected with $2.5 \mu \mathrm{g}$ shRNA; ${ }^{*} \mathrm{P}<0.05$ (one-factor analysis of variance); error bar, standard deviation; $\mathrm{N}=3$.

the expression levels of $F z 2$ and cyclin D1 in MKN45 cells were downregulated to $64.9 \pm 15.9 \%(\mathrm{P}<0.05)$ and $56.7 \pm 6.6 \%$ $(\mathrm{P}<0.05)$ of mock-transfected cells, respectively, at $2.5 \mu \mathrm{g} / \mathrm{well}$ Fz2 shRNA (Fig. 4A and B). In the MKN74 cell line, the expression levels of $F z 2$ and cyclin D1 were downregulated to $3.8 \pm 0.4 \%(\mathrm{P}<0.05)$ and $3.7 \pm 0.8 \%(\mathrm{P}<0.05)$ of those in mocktransfected cells, respectively, at $2.5 \mu \mathrm{g} /$ well Fz2 shRNA (Fig. 4C and D).

To address the possibility that shRNA of Fz2 suppressed cell motility, a scratch assay was performed. Following trans- fection, the cell sheet was scratched and distance of migration was measured over a set time length (Fig. 5A-D). Distance of migration was suppressed from $607 \pm 102 \mu \mathrm{m}$ in mock transfected MKN45 cells to $261 \pm 47 \mu \mathrm{m}(\mathrm{P}<0.05)$ at $2.5 \mu \mathrm{g} / \mathrm{well}$ Fz2 shRNA (Fig. 5E). Similarly, the distance of migration was suppressed from $209 \pm 43 \mu \mathrm{m}$ in mock transfected MKN74 cells to $52 \pm 12 \mu \mathrm{m}(\mathrm{P}<0.05)$ at $2.5 \mu \mathrm{g} /$ well Fz2 shRNA (Fig. 5F).

To reveal the mechanism of suppression of cell motility, the expression levels of $M M P 9$, a gene involved in cancer metastasis (21), were quantitated. MMP9 was downregulated to 

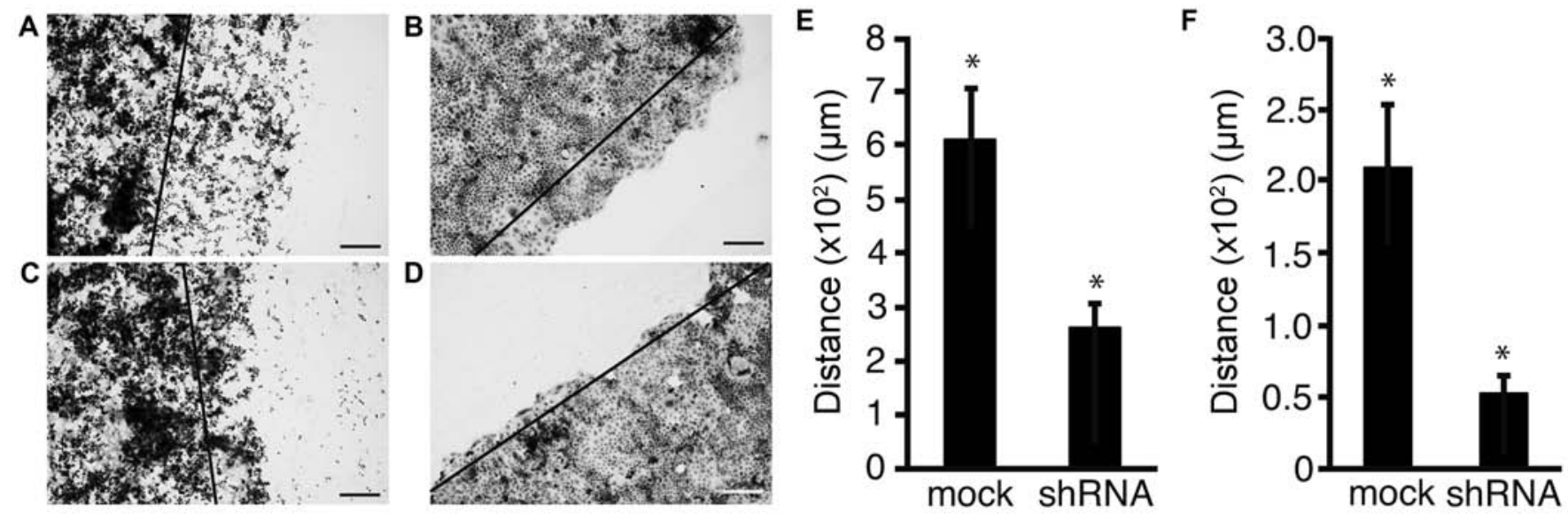

Figure 5. Scratch assay. MKN45 (A and C) or MKN74 cells (B and D) were cultured on chamber slides. The cells were scratched with the tip of a $200-\mu 1$ pipette tip (solid line) and treated with (C and D) or without (A and B) $2.5 \mu \mathrm{g} \mathrm{Fz2} \mathrm{shRNA.} \mathrm{Distance} \mathrm{from} \mathrm{the} \mathrm{scratched} \mathrm{line} \mathrm{to} \mathrm{the} \mathrm{growing} \mathrm{edge} \mathrm{of} \mathrm{the} \mathrm{cells} \mathrm{was}$ measured in MKN45 (E) and MKN74 cells (F). Original magnification, $\mathrm{x} 100$; scale bar, $200 \mu \mathrm{m}$; error bar, standard deviation; ${ }^{*} \mathrm{P}<0.05$; $\mathrm{N}=3$.
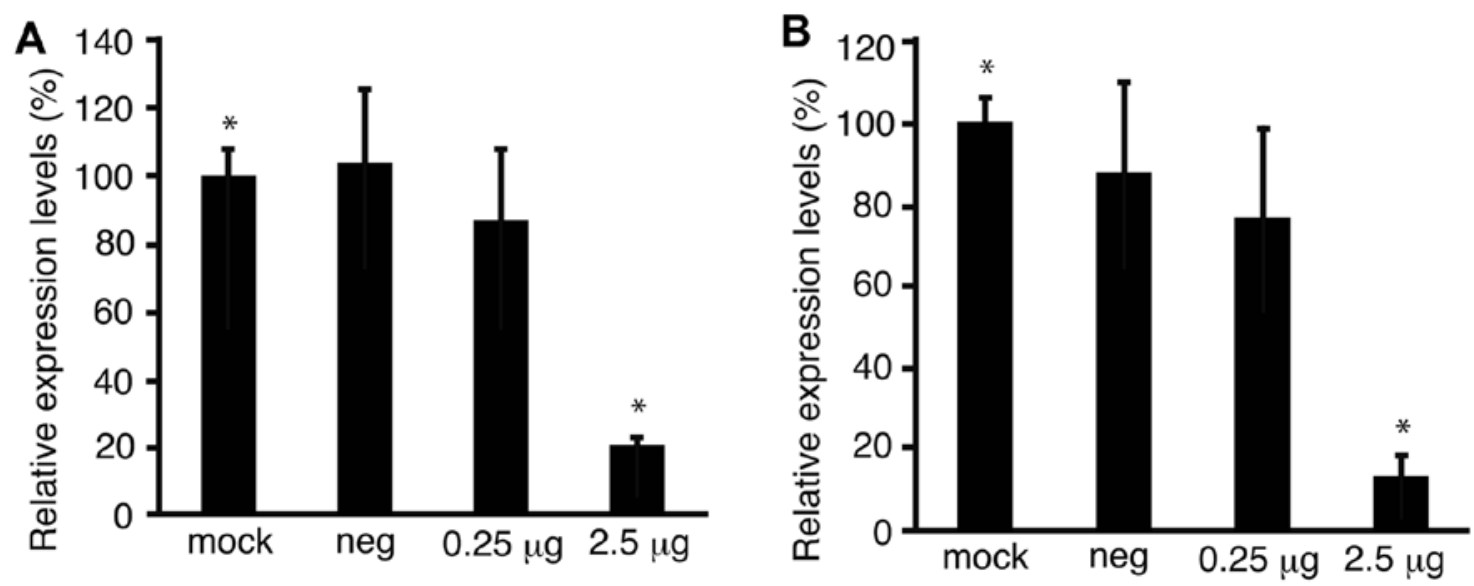

Figure 6. Expression levels of matrix metalloproteinase 9. MKN45 (A) and MKN74 cells (B) were transfected with Fz2 shRNA and subjected to real-time quantitative polymerase chain reaction (PCR) for matrix metalloproteinase 9 . Mock, mock transfected; neg, transfected with negative control; $0.25 \mu \mathrm{g}$, transfected with $0.25 \mu \mathrm{g}$ shRNA; $2.5 \mu \mathrm{g}$, transfected with $2.5 \mu \mathrm{g}$ shRNA; error bar, standard deviation; ${ }^{*} \mathrm{P}<0.05 ; \mathrm{N}=3$.

$20.4 \pm 3.6 \%(\mathrm{P}<0.05)$ and $13.8 \pm 2.8 \%(\mathrm{P}<0.05)$ in MKN45 and MKN74 cells transfected with at $2.5 \mu \mathrm{g}$ Fz2 shRNA, respectively, as compared with mock transfections (Fig. 6A and B).

\section{Discussion}

Expression patterns of $F z$ genes in normal stomach have not been reported (22-29). In our study, $F z 1,2,3,6$ and 8 were shown to be expressed in normal stomach. It has previously been demonstrated that the GC cell lines MKN45 and MKN74 are positive for $F z 3$ expression using northern blot analysis (23). In the present study, we also demonstrated that both cell lines were positive for $F z 3$ expression using RT-PCR. Our data showed that MKN45 and MKN74 cells were negative and positive for $F_{z} 4$ and $F z 6$ expression, respectively, consistent with previous reports $(24,26)$.

In contrast, the present data showed that $F z 2$ was upregulated in the MKN45 GC cell line, but downregulated in MKN74. Fz2 was chosen for further analysis to address the possibility that downregulation of Fz2 might suppress the proliferation and motility of MKN45 and MKN74 cells. MTS and scratch assays clearly showed that Fz2 shRNA successfully suppressed the proliferation and motility of both cell lines. These results indicated that Fz2 might be a novel target for the development of molecular therapies for GC. We also observed Fz2-mediated downregulation of cyclin D1, which is involved in cell proliferation (20) and is also downregulated by an inhibitor of the Wnt pathway (17). These results and our data together suggested that Fz2 shRNA suppressed cell proliferation and motility via suppression of the Wnt pathway.

Our data clearly also demonstrated that $M M P 9$, which has been established to be involved in cell motility (21), was downregulated by Fz2 shRNA in GC cell lines, which also demonstrated suppression of cell motility. As the expression of MMP9 is associated with the expression of Wnt3a in human primary lung cancer tissues (30), this, along with our results, suggested that MMP9 was a downstream target of the Wnt pathway.

One limitation of our study was that Fz2 was expressed in normal stomach. It was difficult to know what types of 
cells were positive for $F z 1,2,3,6$ and 8 . Stomach tissue is composed of mucous, muscle, and serous tissue, as well as nerves and blood vessels.

In conclusion, $F z 2,3,6$ and 8 were expressed in normal stomach, and in MKN45 and MKN74 GC cell lines. Fz2 shRNA suppressed cell proliferation and motility of MKN45 and MKN74 cells, and cyclin D1 and MMP9 expression was downregulated by Fz2 shRNA. Together, these results suggested that Fz2 might be a novel target for the development of molecular therapies for GC.

\section{References}

1. Jemal A, Bray F, Center MM, Ferlay J, Ward E and Forman D: Global cancer statistics. CA Cancer J Clin 61: 69-90, 2011.

2. Kim MY, Cho JH and Cho JY: Ever-changing endoscopic treatment for early gastric cancer: yesterday-today-tomorrow. World J Gastroenterol 20: 13273-13283, 2014.

3. De Vita F, Di Martino N, Fabozzi A, et al: Clinical management of advanced gastric cancer: the role of new molecular drugs. World J Gastroenterol 20: 14537-14558, 2014

4. Gomez-Orte E, Saenz-Narciso B, Moreno S and Cabello J: Multiple functions of the noncanonical Wnt pathway. Trends Genet 29: 545-553, 2013.

5. Tanaka SS, Kojima Y, Yamaguchi YL, Nishinakamura R and Tam PP: Impact of WNT signaling on tissue lineage differentiation in the early mouse embryo. Dev Growth Differ 53: 843-856, 2011

6. MacDonald BT, Tamai K and He X: Wnt/beta-catenin signaling: components, mechanisms, and diseases. Dev Cell 17: 9-26, 2009

7. Takahashi-Yanaga F: Activator or inhibitor? GSK-3 as a new drug target. Biochem Pharmacol 86: 191-199, 2013.

8. Jamieson C, Sharma M and Henderson BR: Targeting the betacatenin nuclear transport pathway in cancer. Semin Cancer Biol 27: 20-29, 2014.

9. Veeck J and Dahl E: Targeting the Wnt pathway in cancer: the emerging role of Dickkopf-3. Biochim Biophys Acta 1825: 18-28, 2012.

10. Katoh M and Katoh M: WNT signaling pathway and stem cell signaling network. Clin Cancer Res 13: 4042-4045, 2007.

11. Qu Y, Dang S and Hou P: Gene methylation in gastric cancer. Clin Chim Acta 424: 53-65, 2013.

12. Hibi K, Sakata M, Yokomizi K, et al: Methylation of the WNT5A gene is frequently detected in early gastric carcinoma. Hepatogastroenterology 59: 2661-2663, 2012.

13. Katoh M: WNT/PCP signaling pathway and human cancer (review). Oncol Rep 14: 1583-1588, 2005.

14. Cui J, Xi H, Cai A, Bian S, Wei B and Chen L: Decreased expression of Sox7 correlates with the upregulation of the Wnt/beta-catenin signaling pathway and the poor survival of gastric cancer patients. Int J Mol Med 34: 197-204, 2014.
15. Yu J, Tao Q, Cheng YY, et al: Promoter methylation of the Wnt/ beta-catenin signaling antagonist Dkk-3 is associated with poor survival in gastric cancer. Cancer 115: 49-60, 2009.

16. Fujimoto T, Tomizawa M and Yokosuka O: SiRNA of frizzled-9 suppresses proliferation and motility of hepatoma cells. Int J Oncol 35: 861-866, 2009.

17. Tomizawa M, Shinozaki F, Motoyoshi Y, et al: Niclosamide suppresses Hepatoma cell proliferation via the Wnt pathway. Onco Targets Ther 6: 1685-1693, 2013.

18. Tomizawa M, Shinozaki F, Sugiyama T, Yamamoto S, Sueishi M and Yoshida T: Frizzled-2: A potential novel target for molecular pancreatic cancer therapy. Oncol Lett 7: 74-78, 2014.

19. Tomizawa M, Shinozaki F, Motoyoshi Y, Sugiyama T, Yamamoto S and Sueishi M: Short hairpin RNA of frizzled-2 suppresses the proliferation of hepatocellular carcinoma cells. Oncol Lett 8: 1519-1522, 2014

20. Casimiro MC, Velasco-Velazquez M, Aguirre-Alvarado C and Pestell RG: Overview of cyclins D1 function in cancer and the CDK inhibitor landscape: past and present. Expert Opin Investig Drugs 23: 295-304, 2014.

21. Vandooren J, Van den Steen PE and Opdenakker G: Biochemistry and molecular biology of gelatinase $\mathrm{B}$ or matrix metalloproteinase-9 (MMP-9): the next decade. Crit Rev Biochem Mol Biol 48: 222-272, 2013

22. Sagara N, Toda G, Hirai M, Terada M and Katoh M: Molecular cloning, differential expression, and chromosomal localization of human frizzled-1, frizzled-2, and frizzled-7. Biochem Biophys Res Commun 252: 117-122, 1998.

23. Kirikoshi H, Koike J, Sagara N, et al: Molecular cloning and genomic structure of human frizzled-3 at chromosome $8 \mathrm{p} 21$. Biochem Biophys Res Commun 271: 8-14, 2000.

24. Kirikoshi H, Sagara N, Koike J, et al: Molecular cloning and characterization of human Frizzled-4 on chromosome 11q14-q21. Biochem Biophys Res Commun 264: 955-961, 1999.

25. Saitoh T, Hirai M and Katoh M: Molecular cloning and characterization of human Frizzled-5 gene on chromosome 2q33.3-q34 region. Int J Oncol 19: 105-110, 2001.

26. Tokuhara M, Hirai M, Atomi Y, Terada M and Katoh M: Molecular cloning of human Frizzled-6. Biochem Biophys Res Commun 243: 622-627, 1998.

27. Saitoh T, Hirai M and Katoh M: Molecular cloning and characterization of human Frizzled-8 gene on chromosome 10p11.2. Int J Oncol 18: 991-996, 2001.

28. Wang YK, Samos CH, Peoples R, Perez-Jurado LA, Nusse R and Francke U: A novel human homologue of the Drosophila frizzled wnt receptor gene binds wingless protein and is in the Williams syndrome deletion at 7q11.23. Hum Mol Genet 6: 465-472, 1997.

29. Koike J, Takagi A, Miwa T, Hirai M, Terada M and Katoh M: Molecular cloning of Frizzled-10, a novel member of the Frizzled gene family. Biochem Biophys Res Commun 262: 39-43, 1999.

30. Lee MA, Park JH, Rhyu SY, Oh ST, Kang WK and Kim HN: Wnt3a expression is associated with MMP-9 expression in primary tumor and metastatic site in recurrent or stage IV colorectal cancer. BMC Cancer 14: 125, 2014. 